\title{
(-) OPEN ACCESS \\ Measuring network disruption in neurodegenerative diseases: New approaches using signal analysis
}

\author{
Roisin McMackin, ${ }^{1}$ Muthuraman Muthuraman, ${ }^{2}$ Sergiu Groppa, ${ }^{2}$ Claudio Babiloni, ${ }^{3,4}$ \\ John-Paul Taylor, ${ }_{1}^{5}$ Matthew C Kiernan, ${ }^{6,7}$ Bahman Nasseroleslami, ${ }^{\oplus}$ \\ Orla Hardiman ${ }^{1,8}$
}

${ }^{1}$ Academic Unit of Neurology, Trinity College Dublin, the University of Dublin, Dublin, Ireland

${ }^{2}$ Department of Neurology, Universitätsmedizin der Johannes Gutenberg-Universität Mainz, Mainz, Germany ${ }^{3}$ Dipartimento di Fisiologia e Farmacologia "Vittorio Erspamer", Università degli Studi di Roma "La Sapienza", Roma, Italy

${ }^{4}$ Istituto di Ricovero e Cura San Raffaele Cassino, Cassino, Italy ${ }^{5}$ Institute of Neuroscience, Newcastle University, Newcastle upon Tyne, UK

${ }^{6}$ Brain \& Mind Centre, University of Sydney, Sydney, Sydney, Australia

${ }^{7}$ Institute of Clinical Neurosciences, Royal Prince Alfred Hospital, Sydney, Sydney, Australia

${ }^{8}$ Beaumont Hospital, Dublin, Ireland

\section{Correspondence to}

Professor Orla Hardiman, Academic Unit of Neurology, Room 5.43, Trinity Biomedical Sciences Institute, 152-160 Pearse Street, Trinity College Dublin, the University of Dublin, Dublin D02 R590, Ireland; hardimao@tcd.ie

Received 23 November 2018 Revised 21 January 2019 Accepted 21 January 2019 Published Online First 13 February 2019

\section{Check for updates}

(c) Author(s) (or their employer(s)) 2019. Re-use permitted under CC BY-NC. No commercial re-use. See rights and permissions. Published by BMJ.

To cite: McMackin $\mathrm{R}$, Muthuraman M, Groppa S, et al. J Neurol Neurosurg Psychiatry

2019:90:1011-1020.

\section{ABSTRACT}

Advanced neuroimaging has increased understanding of the pathogenesis and spread of disease, and offered new therapeutic targets. MRI and positron emission tomography have shown that neurodegenerative diseases including Alzheimer's disease (AD), Lewy body dementia (LBD), Parkinson's disease (PD), frontotemporal dementia (FTD), amyotrophic lateral sclerosis (ALS) and multiple sclerosis (MS) are associated with changes in brain networks. However, the underlying neurophysiological pathways driving pathological processes are poorly defined. The gap between what imaging can discern and underlying pathophysiology can now be addressed by advanced techniques that explore the cortical neural synchronisation, excitability and functional connectivity that underpin cognitive, motor, sensory and other functions. Transcranial magnetic stimulation can show changes in focal excitability in cortical and transcortical motor circuits, while electroencephalography and magnetoencephalography can now record cortical neural synchronisation and connectivity with good temporal and spatial resolution.

Here we reflect on the most promising new approaches to measuring network disruption in $A D, L B D, P D, F T D$, $M S$, and ALS. We consider the most groundbreaking and clinically promising studies in this field. We outline the limitations of these techniques and how they can be tackled and discuss how these novel approaches can assist in clinical trials by predicting and monitoring progression of neurophysiological changes underpinning clinical symptomatology.

\section{INTRODUCTION}

Neurodegenerative diseases including Alzheimer's disease (AD), Lewy body dementia (LBD), Parkinson's disease (PD), frontotemporal dementia (FTD), amyotrophic lateral sclerosis (ALS) and multiple sclerosis (MS) are associated with reproducible neuropathological signatures of neuronal loss, and in most cases, deposition of specific categories of misfolded proteins in anatomic brain regions that correlate with clinical signs. Definitive diagnostic categorisation is correspondingly generally based on clinicopathological correlation, with evidence of characteristic histological changes within specific anatomic regions of the brain. ${ }^{1}$

There is now, however, emerging evidence that the pathogenesis of neurodegeneration is related to widespread and progressive changes in brain networking. This can be defined both in structural terms, as patterns of focal and tract neural degeneration, ${ }^{2}$ and in functional terms, as altered patterns of brain connectivity and neural ${ }^{3}$ and neuromotor ${ }^{4}$ transmission.

Structural neuroimaging including MRI has provided additional information about patterns of grey matter atrophy ${ }^{5}$ and white matter tract degeneration, ${ }^{2}$ while functional MRI and fluorodeoxyglucose positron emission tomography have provided indirect metabolic correlates of network disruption. ${ }^{6}$ These techniques have increased our understanding of the pathogenesis and spread of $\mathrm{AD}, \mathrm{LBD}, \mathrm{PD}$, FTD, ALS and MS, and have offered new therapeutic targets in clinical trials. However, these approaches cannot directly capture abnormal neural transmissions and networking associated with clinical symptoms. This limitation can now be addressed using advanced quantitative electroencephalography and magnetoencephalography (EEG/MEG) and transcranial magnetic stimulation (TMS).

Application of TMS to the motor cortex paired with target muscle electromyography (EMG) can demonstrate changes in excitability in cortical and transcortical motor circuits and offers excellent temporal and good spatial resolution. ${ }^{7}$ By contrast, EEG/MEG has traditionally offered excellent (millisecond) temporal resolution counterbalanced by poor spatial resolution and excessive extracerebral (eg, ocular, head, cardiac) artefacts. ${ }^{8}$ However, the use of EEG/MEG recording systems with a montage of many (up to 256) sensors, removal of artefacts from the digitised signals ${ }^{9}$ and subsequent application of source localisation methods ${ }^{10}$ has substantially increased spatial resolution. Additional quantitative EEG/MEG (qEEG/MEG) methods can now be applied to these high spatial and temporal resolution recordings to generate numerical measures of functional brain activity and functional connectivity between brain areas both at rest ${ }^{11}$ and during specified tasks. ${ }^{12} 13$

These technological improvements have opened exciting opportunities in the application of neurophysiological measurements to provide localised, real-time recordings of neural networking abnormalities in neurodegeneration.

Here we have considered the most promising TMS and EEG/MEG measurements used to investigate AD, LBD, PD, FTD, ALS and MS network pathology. We discuss the neuronal basis of these measurements, describe examples of measurements with potential to enable assessment of early preclinical functional changes associated with neurodegenerative conditions, describe the remaining 
limitations of these technologies and how they can be developed further as inexpensive and informative biomarkers of clinical subphenotype and disease progression.

\section{Electroencephalography and magnetoencephalography}

Electroencephalography (EEG) and magnetoencephalography (MEG) recordings probe (temporal) synchronisation of cortical neuronal activity using sensors placed on (for EEG) or at small distance from (for MEG) the scalp. While the exact mechanisms of the cortical signal generation remains to be understood, there is evidence that scalp-recorded EEG/MEG signals reflect the spatial summation of relatively long-lasting (ten to hundreds of milliseconds) excitatory/inhibitory post-synaptic potentials and dendritic influences of neurons (e.g. the cortical pyramidal neurons), summed together in adjacent regions. ${ }^{14}$ In addition to these post-synaptic potentials the EEG/MEG oscillations also originate from the flow of the activity volleys in the longer-range pathways such as thalamo-cortical connections and loops. ${ }^{15} 16$ While the fast spiking activity of the cortical neurons are not usually considered to be reflected in EEG/MEG signals due to poor spatial summation processes at a given instant, there is evidence that such activities may also appear at higher EEG/ MEG frequencies in the high gamma band $(>40 \mathrm{~Hz}){ }^{17}$

EEG can capture activity in cortical sources oriented both tangentially and radially to the scalp surface due to the electric field propagation and electrical conduction in the volume. However, the signals are attenuated by propagation of electric currents through regions of the head with different resistance. By contrast, while MEG signals are captured exclusively by cortical sources oriented tangentially to the scalp surface due to magnetic field propagation, they are not affected by the propagation of electric currents through head tissues with different resistance. ${ }^{13}$

Two main classes of EEG/MEG signals can be derived from experimental recordings. The first class is event-related with most frequent applications developed in time domain. Within this line are evoked or event-related potentials (EPs/ERPs) and the MEG counterparts, evoked or event-related magnetic fields (EFs/ERFs). EPs/EFs are obtained by the average of hundreds/thousands of short EEG/MEG periods (ten to several hundreds of milliseconds) of activity recorded during repeated sensory stimuli (eg, visual, auditory) with no response required. ${ }^{18}$ Likewise, ERPs/ERFs are obtained by the average of many EEG/MEG periods (ten to several hundreds of milliseconds) of activity, however, they are recorded during repeated sensory stimuli and/or voluntary tasks associated with cognitive processes and usually a behavioural response. ${ }^{19}$ The analysis of those potentials is typically performed in the time domain, investigating latency and amplitude of a sequence of EEG voltage or magnetic field peaks and the underlying cortical source activity. However, more recent applications are developed by a spectral analysis of oscillatory components at delta, theta, alpha, beta and gamma bands from EPs/EFs and ERPs/ERFs. ${ }^{20}$ Finally, less frequent in the field of neurodegenerative diseases, ongoing EEG and MEG rhythms related to sensory and cognitive motor events are analysed with spectral analysis as "event-related desynchronisation/synchronisation" of those frequency bands. ${ }^{21}$

The second class is that of "resting-state" EEG/MEG signals collected in the absence of event, typically analysed in frequency domain (figure 1). This analysis can be performed by linear (eg, discrete Fourier transformation) or nonlinear techniques to quantify brain neural oscillatory activity ${ }^{22}$ in terms of peak frequency, magnitude (eg, power density) and phase, either at sensory or brain source level. Statistical interrelatedness of cortical sources of EEG/MEG signals provides useful information about functional brain connectivity at rest (figure 1) underpinning vigilance, wake-sleep cycle and cognitive functions. ${ }^{23}$ A key benefit of such spectral frequency analysis, particularly at source level, is the ability to relate specific changes in oscillatory EEG/MEG activity (and relative cortical sources) at a given frequency band to specific brain regions, higher functions or neuropathological processes affecting neural or neuromotor transmission. ${ }^{3} 24$

Until recently EEG/MEG recordings have been limited by relatively poor signal-to-noise ratio, excessive artefactual components and low spatial resolution due to a low number of sensors available in acquisition systems. ${ }^{8}$ Those limitations have now been addressed by advanced technologies. Careful application of blind source separation, independent component analyses, and non-parametric statistics ${ }^{25}$ have now dramatically reduced the level of noise and artefact in the EEG/MEG signals. Additionally, advances in cortical source localisation methods, including low resolution electromagnetic brain tomography, and beamforming have improved the accuracy in source estimation of EEG/MEG signals (figure 2), ${ }^{10}$ providing complementary measurements to those derived from functional neuroimaging based on MRI. As a result, abnormalities in such signals due to brain neurodegenerative diseases may be attributed to activation and connectivity in specific cortical (and subcortical) regions. ${ }^{12} 1324$

\section{Transcranial magnetic stimulation}

With the development of coil designs with high focality ${ }^{7}$ and 'threshold tracking' methods which provide excellent intraand inter-day reproducibility, ${ }^{26}$ TMS can now provide robust measures of function in a variety of cortical network components.

By delivering magnetic stimuli to the motor cortex, TMS can invoke muscle responses which are measured by EMG. These responses (motor evoked potentials; MEPs) primarily originate from the activation of the upper motor neurons and related cortical interneurons. The descending pathways activate spinal cord networks including lower motor neurons' connections to associated muscles. The MEP in the target muscles can provide information about the integrity of the corticospinal tract. This is quantified by measures such as the minimum stimulation intensity required to achieve a target MEP amplitude known as motor threshold (figure 3 ). ${ }^{27}$

TMS can also be used to interrogate additional motor pathways of the brain. The use of precisely-timed 'conditioning' magnetic or electrical pulses can activate network components such as interneurons. Measures including short intracortical inhibition (SICI)/long intracortical inhibition (LICI), intracortical-facilitation (ICF), interhemispheric inhibition and facilitation, and short afferent inhibition (SAI)/long afferent inhibition (LAI) can quantify the dysfunction of interneuronal ${ }^{28}$ and callosal ${ }^{29}$ network components and sensorimotor connections, ${ }^{30}$ generating inferences about the excitatory/inhibitory balance across cortical structures and neurotransmitter (e.g. glutamatergic, GABAergic, monoaminergic) function. ${ }^{31}$ This technology has already been commercialised for use in diagnostics and clinical outcome measures for neuromuscular disorders. ${ }^{27}$

TMS has other utilities in interrogating cortical network excitability. Conditioning sub- and supra-threshold stimuli over non-primary motor areas, such as the supplementary motor areas, premotor cortices, dorsolateral prefrontal or posterior parietal regions can reveal the connectivity of these regions to the primary motor cortices and the other brain areas. ${ }^{32}$ In this way, modern TMS can serve much beyond a test for conduction velocity and excitability, but as measures of the integrity and functional connectivity between several cortical regions. ${ }^{27}$ 
A

Time vs. Frequency Domain
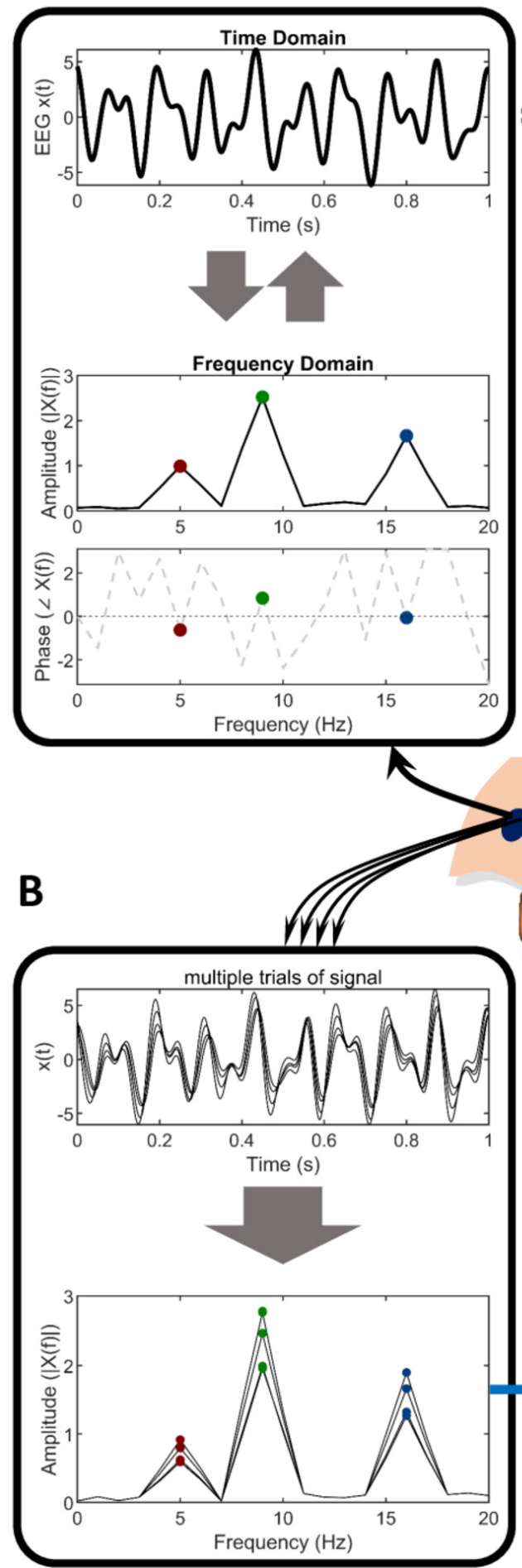

Signal $x$ from Region 1
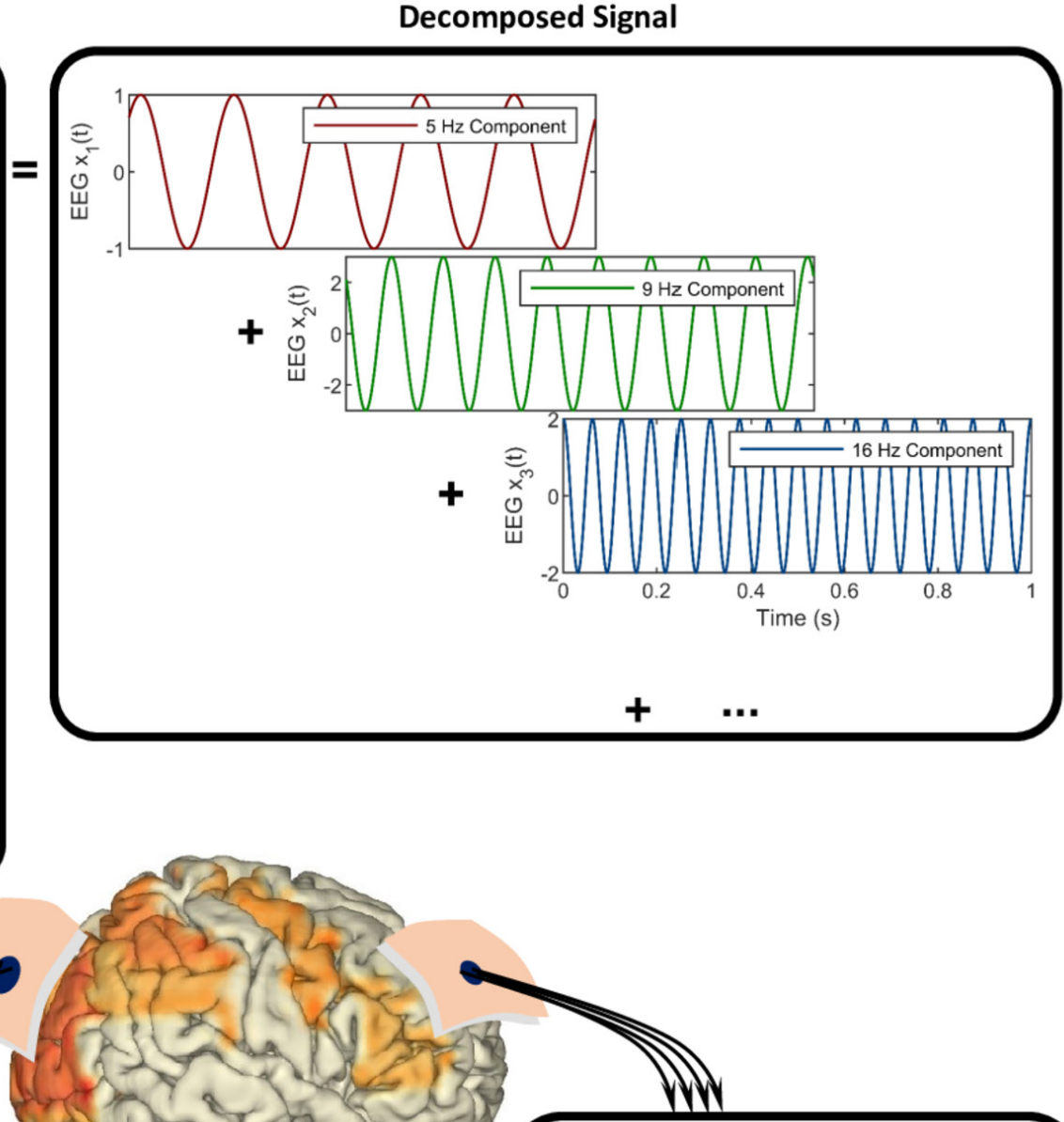

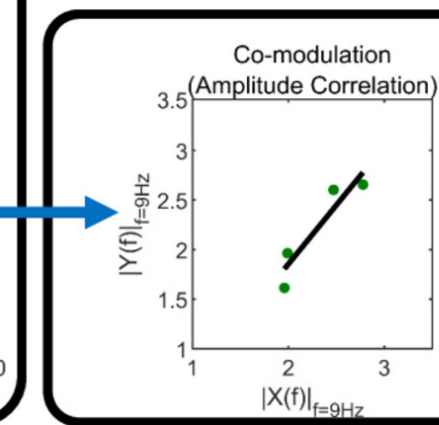

Connectivity bw. Regions 1 \& 2
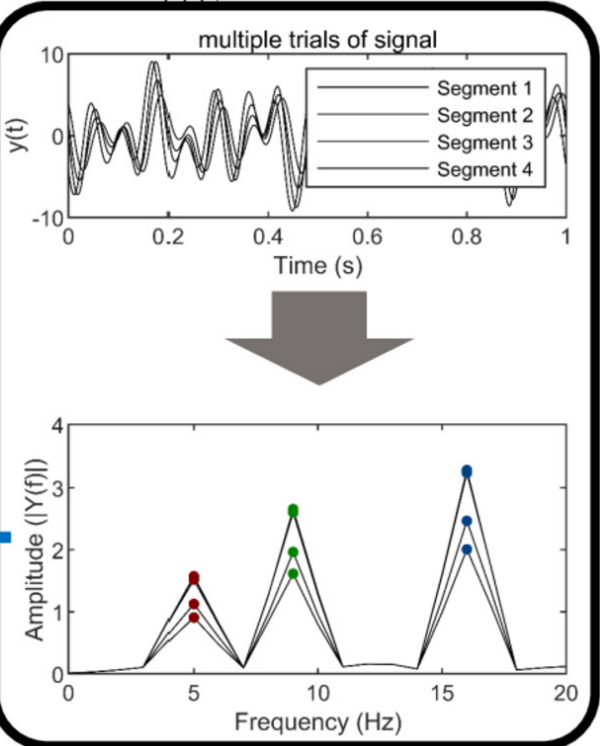

Signal y from Region 2

Figure 1 Schematic of (A) time versus frequency domain representation of electromagnetic neural signals and (B) spectral connectivity between signals from two brain regions. (A) The representation of an exemplary segment of signal as a combination of sinusoidal waveforms. The strengths or amplitudes of the sinusoidal components together with their phase information constitute the frequency domain (spectral) representation of the signal. (B) Multiple epochs, trials or segments of data corresponding to two brain regions can be assessed in specific frequencies to infer and quantify the connectivity.

Interrogation of functional networks in clinical disorders Abnormal brain network function can now be usefully investigated by neurophysiological approaches based on EEG/MEG signals and signals from TMS-linked EMG. These measures are particularly helpful in quantifying signature changes in specific brain regions and in detecting patterns of abnormal 
A

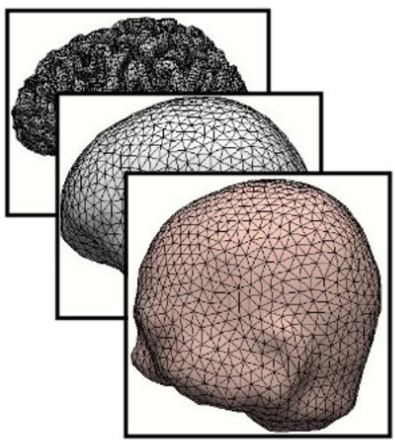

Structural Model (MRI Geometry)

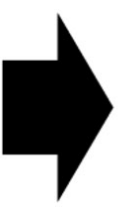

Physical Model
(Electromagnetic Propagation)
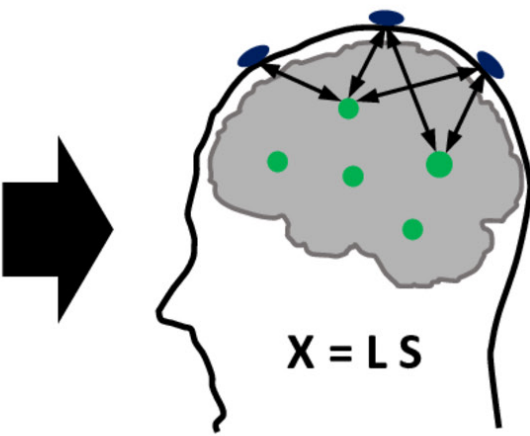

Mathematical Model

(Transformation Equations)

B
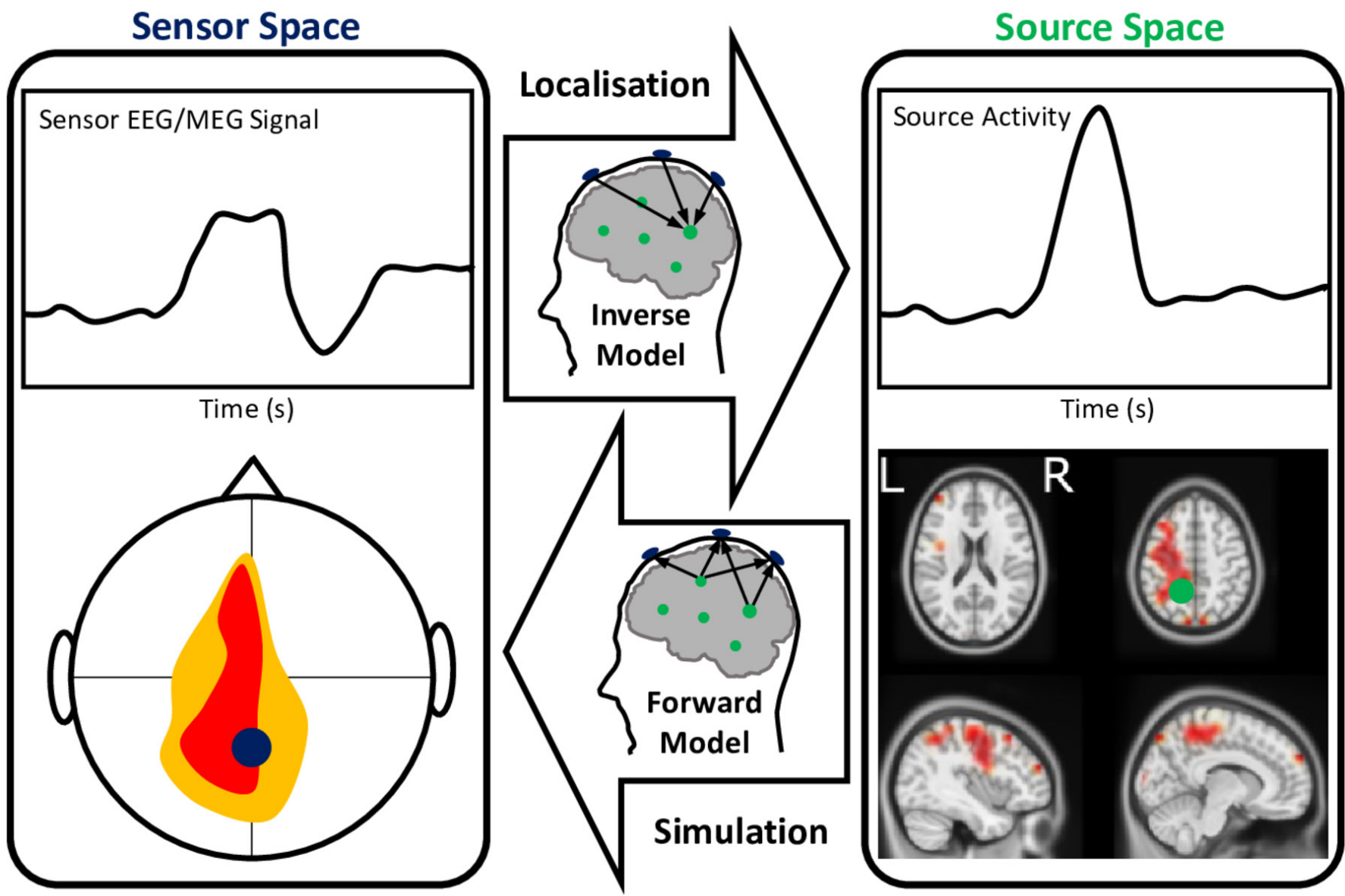

Figure 2 Electromagnetic source imaging using electroencephalography and magnetoencephalography (EEG/MEG) source localisation (A) building models of source and sensor activity and (B) forward versus inverse transformation of signals between the sensors and brain sources. (A) MRI geometry is used for developing structural models of the brain, corticospinal fluid and scalp (among many layers) that are in-between the brain sources generating the neuroelectric activity and electrodes/sensors. The structural model when used together with physical electromagnetic properties of the tissue materials and the governing equations of electromagnetic propagation forms a physical model. The physical model is solved and formulated for the discrete finite number of the modelled sources of activity in the brain (usually about thousands), as well as the EEG/MEG sensors used during the data acquisition (usually a few hundreds). The mathematical model $\mathrm{X}=\mathrm{LS}$ is a multivariate relationship between the sensor activity $(\mathrm{X})$, source activity $(\mathrm{S})$ and the mathematical model $(\mathrm{L})$. (B) This mathematical model can forward-transform the simulated source activities to the sensors, as well as project the recorded sensor activity to localise the underlying brain sources using the constructed inverse model. 
A. Single Pulse TMS

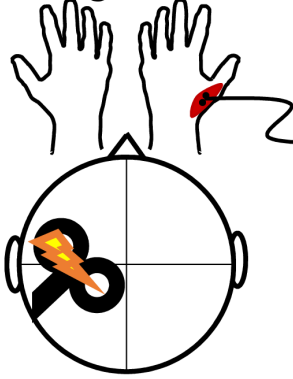

Magnetic pulse artefact Motor evoked potential (MEP)

B. Paired-Pulse TMS

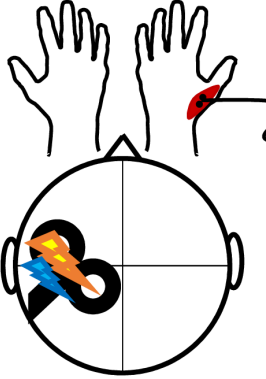

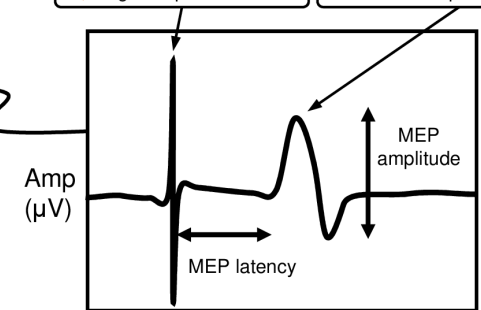

Time (ms)

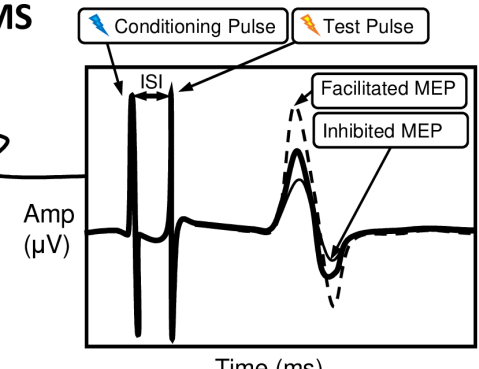

Time (ms)
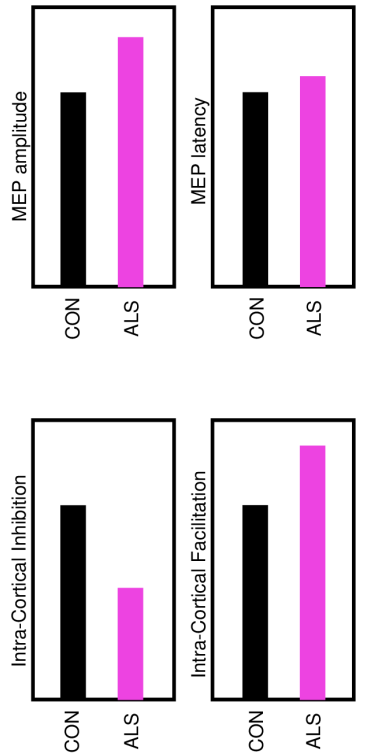

\section{Dual-Coil Paired-Pulse TMS}

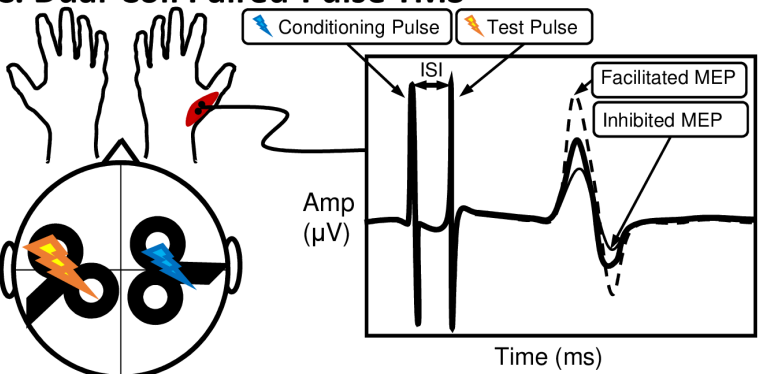

D. Afferent Inhibition Conditioning Pulse Test Pulse

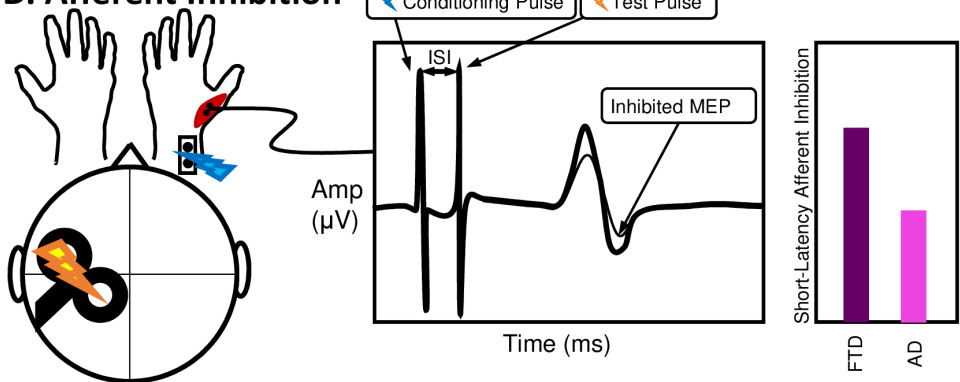

E.

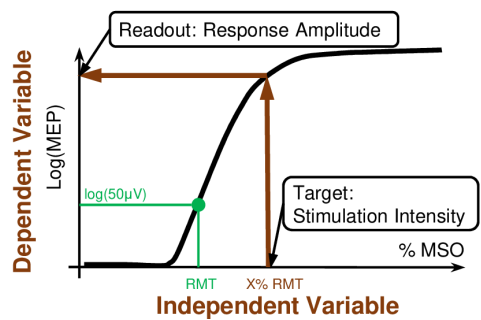

Threshold Tracking TMS

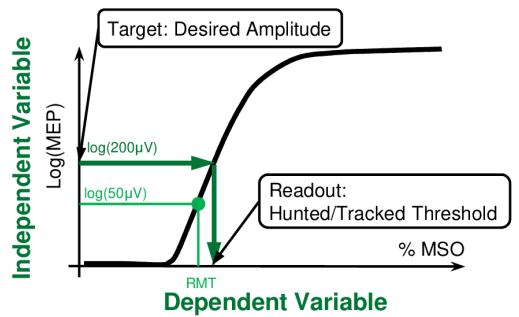

Figure 3 Transcranial magnetic stimulation (TMS) can provide: (A) single-pulse measures, (B) paired-pulse measures, (C) dual-coil paired pulse measures and (D) afferent inhibition measures that when combined with (E) threshold tracking can quantify network connectivity changes in the motor system. The test pulse generates a motor evoked potential (MEP) whose delay and amplitude can be used for quantifying the excitability and conduction in the motor circuits and pathways. This is usually achieved by conditioning the MEP with sub-maximal or supra-maximal conditioning pulses delivered at specific interstimulus intervals before the main test pulse (either in the same brain region with the same coil, over another brain region with a second coil or by peripheral nerve stimulation). This conditioning may facilitate or inhibit the MEP, depending on the (inter-)neuronal populations engaged in each paradigm, the ISI and stimulation intensities or thresholds used. These measures have been proved useful for diagnosis and response prediction in neurodegeneration, eg, in $\mathrm{ALS}^{4}$ and $A D{ }^{30}$ The threshold tracking method is used to achieve a less variable quantification of the stimulus-response characteristics by targeting a specific desired amplitude rather than a specific stimulation intensity. AD, Alzheimer's disease; ALS, amyotrophic lateral sclerosis; MSO, maximum stimulator output; ISI, inter-stimulus interval. 
Table 1 Established neurophysiological changes in neurodegeneration, their clinical utility and discrimination ability

\begin{tabular}{|c|c|c|c|c|c|}
\hline Neurodegeneration & Method & $\begin{array}{l}\text { Neurophysiological } \\
\text { change }\end{array}$ & Clinical application & $\begin{array}{l}\text { Discrimination } \\
\text { statistics }\end{array}$ & References \\
\hline $\begin{array}{l}\text { Alzheimer's disease } \\
\text { Dementia with Lewy bodies }\end{array}$ & $\begin{array}{l}\text { EEG/ } \\
\text { MEG }\end{array}$ & $\begin{array}{l}\quad \downarrow \text { Posterior } \alpha \text { power } \\
\quad \uparrow \text { Parietal } \delta \text { and } \theta \\
\text { power }\end{array}$ & $\begin{array}{l}\text { Prodromal differential } \\
\text { diagnosis } \\
\text { Diagnostic biomarker } \\
\text { Differential diagnosis of AD } \\
\text { and DLB }\end{array}$ & $\begin{array}{l}\text { Sensitivity }-78.3 \% \\
\text { Specificity }-66.7 \% \\
\text { AUROC }-72 \% \\
\text { AUROC }=0.97(\log \delta) \text { and } 0.93 \\
(\log \theta) \\
\text { AUROC }=0.879(\log \delta) \text { and } 0.75 \\
(\log \theta)\end{array}$ & $\begin{array}{l}\text { Andersson et al, Babiloni } \\
\text { et } a^{36}{ }^{63}\end{array}$ \\
\hline Frontotemporal dementia & EEG & $\begin{array}{l}\text { Combined } 25 \text { rsEEG } \\
\text { measures }\end{array}$ & $\begin{array}{l}\text { Differential diagnosis between } \\
\text { ADD, PDD, DLB and bvFTD }\end{array}$ & $\begin{array}{l}\text { AUROC }=100 \% \\
\text { Specificity }=100 \% \\
\text { Selectivity }=100 \%\end{array}$ & Garn et al ${ }^{55}$ \\
\hline Parkinson's disease & EEG & $\begin{array}{l}-\uparrow \beta \text { power } \\
\uparrow 300 \mathrm{~Hz} \text { power }\end{array}$ & $\begin{array}{l}\text { Differential diagnosis } \\
\text { Thresholding of DBS } \\
\text { CT antikinetic measure } \\
\text { CT prokinetic outcome } \\
\text { measure }\end{array}$ & To be determined. & $\begin{array}{l}\text { Assenza et al, } \\
\text { Little et al, } \\
\text { Muthuraman et al, } \\
\text { Assenza et al }{ }^{4852}\end{array}$ \\
\hline Amyotrophic lateral sclerosis & TMS & $\begin{array}{l}\downarrow \text { IShort intracortical } \\
\text { inhibition }\end{array}$ & $\begin{array}{l}\text { Differential diagnosis from } \\
\text { mimic disorders } \\
\text { Prodromal biomarker } \\
\text { CT outcome measure }\end{array}$ & $\begin{array}{l}\text { Sensitivity - } 73.21 \% \\
\text { Specificity - } 80.88 \%\end{array}$ & $\begin{array}{l}\text { Vucic et al, Menon et al, } \\
\text { NCT02450552, } \\
\text { NCT02781454 }\end{array}$ \\
\hline Multiple sclerosis & $\mathrm{EEG}+\mathrm{TMS}$ & - Multimodal ERPs & $\begin{array}{l}\text { Prognosis } \\
\text { CT outcome measure }\end{array}$ & $\begin{array}{l}\text { Sensitivity - } 56.7 \% \\
\text { Specificity - } 88.3 \% \\
\text { Positive predictive value - } \\
70.8 \%\end{array}$ & $\begin{array}{l}\text { Giffroy et al, } \\
\text { NCT01765361 }\end{array}$ \\
\hline
\end{tabular}

ADD, Alzheimer's disease dementia; AUROC, area under the receiver operating characteristics curve; CT, clinical trial; DLB, dementia with Lewy bodies; ERP, event-related potential; PDD, Parkinson's disease dementia; bvFTD, behavioural variant frontotemporal dementia; rsEEG, resting state electroencephalography.

communication between them. Recognising and defining these patterns can thus inform both the nature of the network impairment in neurodegeneration and its progression over time. They can also proffer quantitative biomarkers of these syndromes to improve diagnosis, differential diagnosis, prognosis and therapeutic testing (table 1). The combination of advanced neurophysiological measures into multidimensional markers can further assist in distinguishing neurodegenerative conditions (eg, AD vs $\mathrm{FTD}^{33}$ ) whose affected networks overlap, but also differ. Substantial progress has already been made in these areas, as outlined below for each of the major neurodegenerative conditions.

\section{Mild cognitive impairment and dementias}

Alzheimer's disease dementia (AD) accounts for $50 \%-75 \%$ of dementias, while dementia with Lewy bodies (DLB) accounts for an additional $10 \%-20 \%{ }^{34}$ of all dementias.

Candidate biomarkers of AD and DLB at clinical (dementia) or prodromal (mild cognitive impairment: $\mathrm{MCI}$ ) stages have been generated using frequency domain spectral analysis from eyes-closed resting state (rs) EEG/MEG. ${ }^{35}$ Such rsEEG/rsMEG markers are cost-effective, non-invasive, suitable in cognitively/ physically disabled patients and not susceptible to learning effects. Resting state cortical delta and alpha rhythms in particular reveal compromised network synchronisation and connectivity in AD, Parkinson's disease with dementia (PDD), and DLB at both group and individual levels. ${ }^{3637}$

Impaired cortical neural synchronisation in AD and DLB is quantified by reduced posterior cortical alpha $(8-12 \mathrm{~Hz})$ and beta $(13-30 \mathrm{~Hz})$ rsEEG rhythm intensity and diffuse increases in the intensity of cortical delta $(<4 \mathrm{~Hz})$ and theta $(4-7 \mathrm{~Hz})$ oscillations. ${ }^{38}$ These patterns are more marked in DLB. ${ }^{36}$ Reduced cortical alpha is also found in PDD but to a lesser degree, while widespread theta rhythms are greater in PDD than ADD. ${ }^{37}$ These rsEEG findings have been cross-validated by rsMEG. ${ }^{39-41}$
The clinical importance of these measures is reflected in their presence in MCI stages of degeneration, ${ }^{42}$ their ability to predict progression from MCI to dementia ${ }^{38}$ and their power to discriminate between dementias ${ }^{36}$ and prodromal MCIs ${ }^{43}$ (table 1).

Spectral coherence provides information about cortical network connectivity, which reflects the functional coordination of neural activity. Coherence quantifies the temporal relationship of oscillations in different areas of the brain. This can be measured from rsEEG/rsMEG signals at sensor and cortical source level. In $\mathrm{AD}$, rsEEG coherence is consistently reduced in alpha rhythms. Inconsistent abnormalities in coherence have been reported between electrode pairs in delta and theta rhythms. ${ }^{38}$

These techniques are already in development to measure decline in cognitive status in $\mathrm{ADMCI}^{44}$ using progressive decrement in frontoparietal alpha rhythm synchronisation. These advances demonstrate the utility of neurophysiologic instruments as non-invasive quantitative biomarkers of clinical progression in early AD.

\section{Parkinson's disease}

Parkinson's disease (PD) is characterised by progressive degeneration of dopaminergic neurons of the substantia nigra pars compacta $(\mathrm{SNpc}){ }^{45}$ The high spatial resolution conferred by source localisation of EEG has established that PD network pathology also extends beyond the basal ganglia, including premotor, supplementary motor regions and cerebellar sources. ${ }^{12}$

Degeneration of the $\mathrm{SNpc}$ in $\mathrm{PD}$ prevents the intricate basal ganglia network of feedforward and feedback loops which regulate cortical motor output. ${ }^{45}$ The dysfunction of this network is captured using spectral EEG by an increase in basal ganglia-cortical beta power, as these oscillations represent the probability of a voluntary movement. ${ }^{46}$ Beta oscillations increase longitudinally ${ }^{47}$ and differentiate PD from trends in dementia and stroke. ${ }^{48}$ Causation between these oscillations and PD motor 
symptoms has been established using deep brain stimulation (DBS), as stimulation at $5-20 \mathrm{~Hz}$ (but not $30-50 \mathrm{~Hz}$ ) exacerbates bradykinesia. By contrast, levodopa and DBS of the subthalamic nucleus reduce beta power, correlating with a therapeutic effects on bradykinesia and rigidity. ${ }^{46}$ Levodopa and apomorphine treatment also elicits high gamma $(300 \mathrm{~Hz})$ oscillations. This prokinetic switch from low to high frequency oscillation suggests a clinical utility for excitatory DBS in addition to current inhibitory protocol. ${ }^{48}$

In PD, TMS can detect a reduction in resting motor threshold (RMT) that correlates with poorer Unified Parkinson's Disease Rating Scale motor score, indicative of pathological corticospinal hyperexcitability. ${ }^{50}$ Additional TMS studies demonstrate reduced SICI and increased ICF in the off state of PD, pointing to additional abnormalities in intracortical inhibitory and facilitatory cortical network activity ${ }^{51}$ as drivers of motor hyperexcitability.

These neurophysiologic measures not only provide information about location and nature of PD pathology, but are also important as potential clinical biomarkers. For example, change in subthalamic nucleus beta power can be used to determine the parameters for closed-loop DBS. Stimulation when beta oscillations rise above a threshold is found to provide better therapeutic effect than continuous or random-intermittent delivery of DBS. ${ }^{52}$

Although further studies are required to determine the diagnostic accuracy and longitudinal change of single and combined rsEEG measures in PD, existing TMS, MEG and EEG based evidence has already pointed to the utility of advanced neurophysiological tools as quantitative measures of networks dysfunction.

\section{Frontotemporal dementia}

The well-established dysfunction of prefrontal and temporal networks in FTD can be discriminated by spectral EEG measures, providing a basis for the use of EEG in developing quantitative FTD biomarkers. For example, intrahemispheric information sharing is significantly lower between frontal and temporal areas in behavioural variant FTD (bvFTD). ${ }^{53}$ Source localisation of EEG further demonstrates changes in the orbitofrontal and temporal cortices with enhanced spatial resolution. ${ }^{54}$ While such measures individually have not demonstrated sufficient specificity for use as diagnostic biomarkers alone, the combination of spectral and behavioural measures can reliably discriminate FTD from other types of dementia. For example, a logistic regression model of delta and theta oscillatory activity, combined with cognitive task performance, has demonstrated $93.3 \%$ classification accuracy in differentiating AD and FTD patients. ${ }^{33} \mathrm{~A}$ separate study, combining neuropsychological parameters with frontoparietal and frontotemporal EEG functional connectivity measures from multiple electrodes, similarly achieved an $87.4 \%$ accuracy in discriminating bvFTD patients from controls. ${ }^{53}$

A recent study expanded this approach, using 25 EEG parameters to train support vector machine classifiers to distinguish between AD, PD or LBD and bvFTD, such that $100 \%$ specificity, sensitivity and accuracy was achieved. ${ }^{55}$

The use of such parameters in differential diagnosis has also recently been expanded beyond the dementias, to explore the differentiation of psychiatric and neurodegenerative disorders which overlap clinically. For example, a combination of EEG and imaging parameters has demonstrated $87 \%$ accuracy in differentiation of FTD from late-onset bipolar disorder which have overlapping behavioural symptoms. ${ }^{56}$ Such distinction based on network pathology may assist in distinction of solely psychiatric disorders from early manifestations of neurodegeneration. ${ }^{57}$

\section{Amyotrophic lateral sclerosis}

Amyotrophic lateral sclerosis (ALS) is characterised by progressive degeneration of upper and lower motor neurons. ${ }^{58}$ Diagnostic delays of up to 15 months from symptom onset are common in ALS. Access to clinical trials at an early stage of disease is accordingly compromised. ${ }^{59}$

Quantitative measures of brain network dysfunction have the capacity to reduce diagnostic delay and improve diagnostic accuracy. However, while imaging and physiological studies have demonstrated broader patterns of atrophy across ALS, such as in the thalamus, ${ }^{60}$ basal ganglia ${ }^{5}$ and prefrontal cortex, ${ }^{6162}$ resting state EEG/MEG in ALS can reliably identify increases in intraand inter-motor cortical functional connectivity. ${ }^{34}$ This finding is consistent with evidence of motor hyperexcitability as defined by threshold tracking TMS in ALS.

Using TMS, hyperexcitability in ALS is quantified by reduced motor threshold, a measure of corticospinal excitability. SICI, a measure of GABAergic interneuron function, is also consistently reduced in ALS, implying loss of inhibition as a source of network hyperexcitability. ${ }^{63}$ This decrease in SICI can distinguish ALS from mimic neuromuscular syndromes with $73 \cdot 21 \%$ sensitivity and $80 \cdot 88 \%$ specificity. ${ }^{28}$ Decreased SICI may also be present pre-symptomatically, ${ }^{4}$ providing a prodromal biomarker.

The most common extra-motor impairment in ALS is executive dysfunction, occurring in up to $50 \%$ of patients. ${ }^{64} 65 \mathrm{In}$ addition to the existence of cognitively impaired (and non-impaired) patient groups ${ }^{64}$ the existence of cognitive ALS subphenotypes is supported by a $14.3 \%$ genetic correlation between schizophrenia ${ }^{66}$ and ALS and the increased risk of psychiatric disorders, FTD and ALS conveyed by C9orf72 expansions. ${ }^{67}$

Biomarkers of such cognitive network impairment are urgently required for early discrimination of this subphenotype, in addition to providing outcome measures for cognition-targeting clinical trials. As cognitive impairment in ALS correlates with poorer prognosis, ${ }^{64}$ subphenotyping based on domains of network impairment has the potential to improve early prognostic accuracy and facilitate recruitment to cognition-targeted therapeutic trials.

Promising work using cognitive ERPs can capture millisecond-by-millisecond differences in executive network activity. Application of quantitative EEG analysis of ERP in ALS patients has shown increased average delay in the mismatch negativity ERP, a measure of involuntary attention switching. This correlates to Stroop task performance, a psychological test of inhibitory control and attention shifting. ${ }^{19}$ On source localisation of this EEG signal it is revealed that during this task ALS patients demonstrate excessive left frontoparietal activity which correlates with poorer inhibitory control, in addition to decreased bilateral inferior frontal activity. ${ }^{12}$ Such measures have the advantage of providing an objective and quantitative biomarkers of cognitive network dysfunction that are not biassed by learning effects or physical disability.

As demonstrated for dementias, combination of such measures of motor, cognitive and broader network dysfunction could further enhance discrimination ability from mimic neuromuscular disorders, providing a non-invasive, low-cost, multidimensional biomarker of ALS, in addition to characterising ALS subphenotypes. 


\section{Multiple sclerosis}

Multiple sclerosis (MS) is a disorder characterised by foci of inflammation and demyelination through the central nervous system, leading to axon loss and grey matter generation ${ }^{68}$ with clinical evidence of progressive neurodegeneration. The clinical use of somatosensory, motor and visual evoked potentials to document silent and manifest lesions in MS was commonplace before replacement with MRI scanning. ${ }^{69}$

Neurophysiological tools are now used to objectively characterise connectivity by MEG and EEG measures, such as resting-state network microstate activity measures, which predict total disease duration, annual relapse rate, disability score, depression score and cognitive fatigue measures in relapse-remitting MS. $^{70}$

Advances in event-related EEG/MEG parameters has also captured specific network dysfunction underlying fatigue in MS, with increased P100 visual ERP latency correlating with visual fatigue scores and increased latency in the $\mathrm{V}$ component of brainstem auditory ERPs correlating with brainstem fatigue scores. ${ }^{71}$ Changes in TMS-evoked motor potentials and centralmotor conduction time also show strong correlation to disability score. $^{72}$

These TMS and EEG parameters are now re-emerging as important MS biomarkers, with the realisation that MRI measures can show weak association with clinical presentation, known as the clinical/MRI paradox. ${ }^{73}$ A combination of electrophysiological parameters, labelled the 'global evoked potential' score, predicts both annual disability score progression and risk of disability progression and a cut-off has been defined to detect patients at high risk of disability progression at a predictive value of $70 \% .^{18}$

\section{Neurophysiologic signal analysis as biomarkers and outcome measures in clinical trials}

For diagnostic biomarkers, mathematically-defined combinations of neurophysiology based network measures are likely to evolve over the coming years. For prognostic and stratification biomarkers, however, the neurophysiological validity and relevance of the network activity is the priority to assure meaningful interpretations.

Standardisation of biomarkers based on neural signals is already well advanced for neuropsychiatric disorders, ${ }^{74}$ but have not yet been used consistently in neurodegenerative conditions, despite the acknowledged biological overlap between conditions such as ALS and schizophrenia. ${ }^{66}$ Biomarker development in neurodegeneration is promising, although verification and validation of the sensitivity and specificity reported for these biomarkers (table 1) will be required within each neurodegenerative disease.

The clinical utility of EEG in resting-state has already been used as a pharmacodynamic marker in a dose-finding study in rodents, and has been successfully translated to a human study of an antidepressant compound. ${ }^{76}$ Similarly, a recent retigabine trial in ALS has used the decrease in SICI as a recruitment criterion (NCT02450552) while a trial of mexiletine (NCT02781454) is now using change in RMT and SICI as primary and secondary outcome measures respectively. SAI has also shown promise as a predictor of cholinesterase inhibitor response in $\mathrm{AD},{ }^{30}$ warranting further investigation as an electrophysiological outcome measure.

Resting-state EEG has also been utilised as a secondary outcome measure in testing the nutritional aid Souvenaid as a therapy in $\mathrm{AD}$, with change in delta band functional connectivity showing improved trajectory. ${ }^{77}$ A combination of multimodal evoked potentials was also used an outcome measure in a phase III trial (NCT01765361) of the recently approved drug ocrelizumab for MS.

These early studies point to a move towards therapies based on modulation of network dysfunction, allowing for earlier, and possibly presymptomatic intervention based on early changes in physiological measures. ${ }^{4}$

\section{CURRENT LIMITATIONS}

While consistent changes in electrophysiological measures have been identified in some neurodegenerative diseases (such as increased beta power in $\mathrm{PD}^{48} 4952$ ), others require further evaluation and validation (eg, increased motor threshold in Huntington's disease or decreased motor threshold in $\mathrm{PD}^{78}$ ). Furthermore, while single measures may for some diseases provide sufficient specificity and selectivity for diagnosis, such as SICI in ALS, ${ }^{48} 63$ this has yet to be achieved for other diseases such as AD. ${ }^{79}$ In the longer term, it is likely that combinations of multiple measures (including neurophysiological, behavioural and neuroimaging) will be required to capture broader patterns of network disruption. Individual measures are more likely to provide tools for differential diagnosis to enhance patient stratification and provide quantitative measures in clinical trials. ${ }^{79}$ Going forward, large multicentre studies will be required to validate all individual and multidimensional physiological measures for use in routine clinical settings.

\section{CONCLUSION AND FUTURE DIRECTIONS}

The use of physiological methods such as EEG, MEG and TMS permits the interrogation of network function with excellent temporal resolution. These methods are non-invasive, do not depend on intact motor or language function or participant engagement, and are substantially lower in cost, compared with neuroimaging alternatives using MRI or positron emission tomography. With the evolution of EEG/MEG recording techniques, improved preliminary data processing, source estimation techniques and focal TMS coils, the perceived limitations of excess noise and poor spatial resolution have been substantially reduced. As a result, the location, nature and overlap of cortical neural network disruption in the brain neurodegenerative diseases can be directly measured and quantified as a readout reflecting cortical neural excitability, synchronisation and connectivity as relevant underpinning of cognitive and sensorimotor functions.

While underutilised in multicentric clinical trials testing disease-modifying drugs for major neurodegenerative diseases to date, these improved quantitative neurophysiological measures have enhanced the state-of-the-art in clinical neurophysiology research. They have shown that neurodegenerative diseases affect the cortical neural excitability, synchronisation and functional connectivity in relation to cognitive, sensory and motor impairment in AD, DLB, PD, FTD, ALS and MS patients. Future longitudinal, harmonised, multi-centre, cross-validation studies will provide a non-invasive quantification of cortical neural network disruption in the neurodegenerative diseases and can provide neurophysiological predictors of clinical trajectories and correlates of disease progression.

Important future directions will also include the application of such biomarkers for earlier and possibly pre-symptomatic intervention, enhancing the probability of therapeutic success; and the development of physiological parameters as objective, numerical outcome measures that provide sensitive non-invasive 
and cost effective tools for detecting therapeutic effects of new medicinal compounds.

\section{SELECTION CRITERIA}

References were accessed using the NCBI Pubmed, Embase and Web of Science databases.

Search criteria used for review of each neurodegenerative disease included combination of methodology terms ("EEG", "evoked potential", "event related potential”, "MEG", "transcranial magnetic stimulation", "TMS", "deep brain stimulation") with disease name terms ("Alzheimers", "Parkinsons", "Parkinsonism", "frontotemporal dementia", "amyotrophic lateral sclerosis", "multiple sclerosis", "Lewy bodies") in the previous 15 years 2003 to 2018. Papers were chosen so as to highlight those with the best methodology (number of subjects and patients, diagnosis based on biomarkers in line with the international Guidelines, use of advanced EEG/MEG/TMS techniques at the state-of-the art, research groups with high reputation) investigating physiological measures with potential clinical utility. The number of papers accessed per database, search criterion and disease reviewed, is summarised in online supplementary table 1.

Reviews highlighting the limitations of the methods reviewed in the clinical application were also discussed to provide counter-information in order to minimise potential bias. Search criteria for review of the remaining limitations included combination of methodology terms ("EEG", "MEG", "transcranial magnetic stimulation", "TMS") with the word "problem" or "limitations" and "neurodegenerative" in the previous 5 years 2013 to 2018, so as to restrict review of these limitations to those applicable to the current state of the art.

Contributors $\mathrm{OH}$ planned the review, convened the expert group, oversaw the project development and had final editorial oversight of the manuscript. $\mathrm{OH}$ and RM wrote the introduction, limitations and conclusions. BN, MM, SG and RM wrote the methodology section. All authors reviewed and reported on neurodegenerative disease literature. BN composed the figures. RM composed the tables. All authors edited and revised the manuscript prior to submission. $\mathrm{OH}$ and RM submitted the study.

Funding $\mathrm{OH}, \mathrm{BN}$ and $\mathrm{RM}$ were supported by the Irish Health Research Board [grant numbers: EIA-2017-019, HRB-MRCG-2018-02, HRB-JPND-2017-1], Science Foundation Ireland [grant number: 16/ERCD/3854], the Irish Research Council [grant numbers: GOIPG/2017/1014,GOIPD/2015/213], the American ALS Association [grant number: ALS18-CM-396] and Research Motor Neurone. JPT was supported by the National Institute for Health Research (NIHR), Newcastle Biomedical Research Centre based at Newcastle upon Tyne Hospitals NHS Foundation Trust and Newcastle University. The views expressed are those of the authors and not necessarily those of the NHS, the NIHR or the Department of Health. MM and SG were supported by Transregional Collaborative Research Center (CRC) SFB TR-128. MCK was supported by the National Health and Medical Research Council of Australia Program Grant [grant number: 1132524] and Practitioner Fellowship [grant number: 1156093]. Funding sources had no involvement in the writing of this review.

Competing interests $\mathrm{OH}$ receives personal fees from Taylor and Francis, Cytokinetics and Wave Pharmaceuticals, outside the submitted work.

Patient consent for publication Not required.

Provenance and peer review Commissioned; externally peer reviewed.

Open access This is an open access article distributed in accordance with the Creative Commons Attribution Non Commercial (CC BY-NC 4.0) license, which permits others to distribute, remix, adapt, build upon this work non-commercially, and license their derivative works on different terms, provided the original work is properly cited, appropriate credit is given, any changes made indicated, and the use is non-commercial. See: http://creativecommons.org/licenses/by-nc/4.0/.

\section{REFERENCES}

1 Love S. Post mortem sampling of the brain and other tissues in neurodegenerative disease. Histopathol 2004:44:309-17.

2 Müller H-P, Kassubek J. Diffusion tensor magnetic resonance imaging in the analysis of neurodegenerative diseases. JoVE;(77).
3 Nasseroleslami B, Dukic S, Broderick M, et al. Characteristic increases in EEG connectivity correlate with changes of structural MRI in amyotrophic lateral sclerosis. Cereb Cortex 2017:1-15.

4 Vucic S, Nicholson GA, Kiernan MC. Cortical hyperexcitability may precede the onset of familial amyotrophic lateral sclerosis. Brain 2008;131:1540-50.

5 Bede $\mathrm{P}$, Elamin M, Byrne $\mathrm{S}$, et al. Basal ganglia involvement in amyotrophic lateral sclerosis. Neurology 2013;81:2107-15.

6 Keppler JS, Conti PS. A cost analysis of positron emission tomography. AJR Am J Roentgenol 2001;177:31-40.

7 Ueno S, Matsuda T, Fujiki M. Functional mapping of the human motor cortex obtained by focal and vectorial magnetic stimulation of the brain. IEEE Trans Magn 1990;26:1539-44.

8 Reis PM, Hebenstreit F, Gabsteiger F, et al. Methodological aspects of EEG and body dynamics measurements during motion. Front Hum Neurosci 2014;8.

9 Mognon A, Jovicich J, Bruzzone L, et al. Adjust: an automatic EEG artifact detector based on the joint use of spatial and temporal features. Psychophysiology 2011:48:229-40.

10 Darvas F, Pantazis D, Kucukaltun-Yildirim E, et al. Mapping human brain function with MEG and EEG: methods and validation. Neuroimage 2004;23(Suppl 1):S289-S299.

11 Muthuraman M, Raethjen J, Koirala N, et al. Cerebello-cortical network fingerprints differ between essential, Parkinson's and mimicked tremors. Brain 2018;141:1770-81.

12 McMackin R, Dukic S, Broderick M, et al. Dysfunction of attention switching networks in amyotrophic lateral sclerosis. Neuroimage Clin 2019;22.

13 Muthuraman $\mathrm{M}$, Hellriegel $\mathrm{H}$, Hoogenboom N, et al. Beamformer source analysis and connectivity on concurrent EEG and MEG data during voluntary movements. PLoS One 2014;9:e91441.

14 Murakami S, Okada Y. Contributions of principal neocortical neurons to magnetoencephalography and electroencephalography signals. J Physiol 2006;575:925-36.

15 Neuper C, Klimesch W. Event-related dynamics of brain oscillations. Elsevier, 2006.

16 Vuckovic A, Hasan MA, Osuagwu B, et al. The influence of central neuropathic pain in paraplegic patients on performance of a motor imagery based brain computer interface. Clin Neurophysiol 2015;126:2170-80.

17 Teleńczuk B, Baker SN, Kempter R, et al. Correlates of a single cortical action potential in the epidural EEG. Neuroimage 2015;109:357-67.

18 Giffroy X, Maes N, Albert A, et al. Multimodal evoked potentials for functional quantification and prognosis in multiple sclerosis. BMC Neurol 2016;16.

19 lyer PM, Mohr K, Broderick M, et al. Mismatch negativity as an indicator of cognitive sub-domain dysfunction in amyotrophic lateral sclerosis. Front Neurol 2017;8.

20 Başar E, Femir B, Emek-Savaş DD, et al. Increased long distance event-related gamma band connectivity in Alzheimer's disease. Neuroimage Clin 2017:14:580-90.

21 Graimann B, Pfurtscheller G. Quantification and visualization of event-related changes in oscillatory brain activity in the time-frequency domain. In: Neuper C, Klimesch W, eds. Progress in brain research. Elsevier, 2006: 79-97.

22 Siegel M, Donner TH, Engel AK. Spectral fingerprints of large-scale neuronal interactions. Nat Rev Neurosci 2012;13:121-34.

23 van Diessen E, Numan T, van Dellen E, et al. Opportunities and methodological challenges in EEG and MEG resting state functional brain network research. Clin Neurophysiol 2015;126:1468-81.

24 Proudfoot M, Colclough GL, Quinn A, et al. Increased cerebral functional connectivity in ALS: a resting-state magnetoencephalography study. Neurology 2018;90.

25 Dukic S, Iyer PM, Mohr K, et al. Estimation of coherence using the median is robust against EEG artefacts. Conf Proc IEEE Eng Med Bio/ Soc 2017;2017:3949-52.

26 Samusyte G, Bostock H, Rothwell J, et al. P157 reliability of threshold tracking technique for short interval intracortical inhibition. Clinical Neurophysiology 2017; 128:e93

27 Groppa S, Oliviero A, Eisen A, et al. A practical guide to diagnostic transcranial magnetic stimulation: report of an IFCN Committee. Clin Neurophysiol 2012;123:858-82.

28 Menon P, Geevasinga N, Yiannikas C, et al. Sensitivity and specificity of threshold tracking transcranial magnetic stimulation for diagnosis of amyotrophic lateral sclerosis: a prospective study. Lancet Neurol 2015;14:478-84.

29 Ibey RJ, Bolton DA, Buick AR, et al. Interhemispheric inhibition of corticospinal projections to forearm muscles. Clin Neurophysiol 2015;126:1934-40.

30 Di Lazzaro V, Oliviero A, Pilato F, et al. Neurophysiological predictors of long term response to AChE inhibitors in AD patients. J Neurol Neurosurg Psychiatry 2005;76:1064-9.

31 Agarwal S, Koch G, Hillis AE, et al. Interrogating cortical function with transcranial magnetic stimulation: insights from neurodegenerative disease and stroke. J Neurol Neurosurg Psychiatry 2019;90:47-57.

32 Ruddy KL, Leemans A, Woolley DG, et al. Structural and functional cortical connectivity mediating cross education of motor function. J. Neurosci. 2017;37:2555-64.

33 Lindau M, Jelic V, Johansson SE, et al. Quantitative EEG abnormalities and cognitive dysfunctions in frontotemporal dementia and Alzheimer's disease. Dement Geriatr Cogn Disord 2003;15:106-14

34 Burns A, lliffe S. Dementia. BMJ 2009;338:b75 
35 Babiloni C, Lizio R, Marzano N, et al. Brain neural synchronization and functional coupling in Alzheimer's disease as revealed by resting state EEG rhythms. Int J Psychophysiol Off J Int Organ Psychophysiol 2016;103:88-102.

36 Andersson M, Hansson O, Minthon L, et al. Electroencephalogram variability in dementia with Lewy bodies, Alzheimer's disease and controls. Dement Geriatr Cogn Disord 2008:26:284-90.

37 Babiloni C, De Pandis MF, Vecchio F, et al. Cortical sources of resting state electroencephalographic rhythms in Parkinson's disease related dementia and Alzheimer's disease. Clinical Neurophysiology 2011;122:2355-64.

38 Vecchio F, Babiloni C, Lizio R, et al. Resting state cortical EEG rhythms in Alzheimer's disease: toward EEG markers for clinical applications: a review. Supp/Clin Neurophysiol 2013;62:223-36.

39 Mandal PK, Banerjee A, Tripathi M, et al. A comprehensive review of magnetoencephalography (MEG) studies for brain functionality in healthy aging and Alzheimer's disease (AD). Front Comput Neurosci 2018;12.

40 Olde Dubbelink KT, Stoffers D, Deijen JB, et al. Cognitive decline in Parkinson's disease is associated with slowing of resting-state brain activity: a longitudinal study. Neurobiol Aging 2013;34:408-18.

41 Franciotti $R$, lacono D, Della Penna $S$, et al. Cortical rhythms reactivity in $A D$, LBD and normal subjects: a quantitative MEG study. Neurobio/ Aging 2006;27:1100-9.

42 Babiloni C, Del Percio C, Lizio R, et al. Abnormalities of cortical neural synchronization mechanisms in subjects with mild cognitive impairment due to Alzheimer's and Parkinson's diseases: an EEG study. J Alzheimers Dis 2017:59:339-58.

43 Babiloni C, Del Percio C, Lizio R, et al. Abnormalities of resting state cortical EEG rhythms in subjects with mild cognitive impairment due to Alzheimer's and Lewy body diseases. J Alzheimers Dis 2018;62:247-68.

44 Babiloni C, Frisoni G, Steriade M, et al. Frontal white matter volume and delta EEG sources negatively correlate in awake subjects with mild cognitive impairment and Alzheimer's disease. Clin Neurophysiol 2006;117:1113-29.

45 Surmeier DJ, Obeso JA, Halliday GM. Selective neuronal vulnerability in Parkinson disease. Nat Rev Neurosci 2017;18:101-13.

46 Jenkinson N, Brown P. New insights into the relationship between dopamine, beta oscillations and motor function. Trends Neurosci 2011;34:611-8.

47 Caviness JN, Hentz JG, Belden CM, et al. Longitudinal EEG changes correlate with cognitive measure deterioration in Parkinson's disease. J Park Dis 2015;5:117-24.

48 Assenza G, Capone F, di Biase L, et al. Corrigendum: oscillatory activities in neurological disorders of elderly: biomarkers to target for neuromodulation. Front Aging Neurosci 2017:9.

49 Muthuraman M, Koirala N, Ciolac D, et al. Deep brain stimulation and L-dopa therapy: concepts of action and clinical applications in Parkinson's disease. Front Neurol 2018;9.

50 Park J, Chang WH, Cho JW, et al. Usefulness of transcranial magnetic stimulation to assess motor function in patients with parkinsonism. Ann Rehabil Med 2016;40:81-7.

$51 \mathrm{Ni} \mathrm{Z}$, Bahl N, Gunraj CA, et al. Increased motor cortical facilitation and decreased inhibition in Parkinson disease. Neurology 2013;80:1746-53.

52 Little S, Pogosyan A, Neal S, et al. Adaptive deep brain stimulation in advanced Parkinson disease. Ann Neurol 2013;74:449-57.

53 Dottori M, Sedeño L, Martorell Caro M, et al. Towards affordable biomarkers of frontotemporal dementia: a classification study via network's information sharing. Sc Rep 2017;7.

54 Nishida K, Yoshimura M, Isotani T, et al. Differences in quantitative EEG between frontotemporal dementia and Alzheimer's disease as revealed by LORETA. Clin Neurophysio/ 2011;122:1718-25.

55 Garn H, Coronel C, Waser M, et al. Differential diagnosis between patients with probable Alzheimer's disease, Parkinson's disease dementia, or dementia with Lewy bodies and frontotemporal dementia, behavioral variant, using quantitative electroencephalographic features. J Neural Transm 2017:124:569-81.
56 Metin SZ, Erguzel TT, Ertan G, et al. The use of quantitative EEG for differentiating frontotemporal dementia from late-onset bipolar disorder. Clin EEG Neurosci 2018;49:171-6.

57 Lanata SC, Miller BL. The behavioural variant frontotemporal dementia (bvFTD) syndrome in psychiatry. J Neurol Neurosurg Psychiatry 2016;87:501-11.

58 Ludolph A, Drory V, Hardiman 0, et al. A revision of the El Escorial criteria - 2015. Amyotroph Lateral Scler Frontotemporal Degener 2015;16:291-2.

59 Galvin M, Ryan P, Maguire $S$, et al. The path to specialist multidisciplinary care in amyotrophic lateral sclerosis: a population- based study of consultations, interventions and costs. Plos One 2017;12.

60 Menke RAL, Proudfoot M, Talbot K, et al. The two-year progression of structural and functional cerebral MRI in amyotrophic lateral sclerosis. Neurolmage: Clinical 2018;17:953-61.

61 Iyer PM, Egan C, Pinto-Grau M, et al. Functional connectivity changes in restingstate EEG as potential biomarker for amyotrophic lateral sclerosis. PLoS One 2015; 10:e0128682

62 Machts J, Cardenas-Blanco A, Acosta-Cabronero J, et al. Prefrontal cortical thickness in motor neuron disease. Neuroimage Clin 2018;18:648-55.

63 Grieve SM, Menon P, Korgaonkar MS, et al. Potential structural and functiona biomarkers of upper motor neuron dysfunction in ALS. Amyotrophic Lateral Sclerosis and Frontotemporal Degeneration 2016;17:85-92.

64 Elamin M, Phukan J, Bede $\mathrm{P}$, et al. Executive dysfunction is a negative prognostic indicator in patients with ALS without dementia. Neurology 2011;76:1263-9.

65 Ringholz GM, Appel SH, Bradshaw M, et al. Prevalence and patterns of cognitive impairment in sporadic ALS. Neurology 2005;65:586-90.

66 McLaughlin RL, Schijven D, van Rheenen W, et al. Genetic correlation between amyotrophic lateral sclerosis and schizophrenia. Nat Commun 2017;8.

67 Devenney EM, Ahmed RM, Halliday G, et al. Psychiatric disorders in C9orf72 kindreds: study of 1,414 family members. Neurology 2018;91:e1498-507.

68 Di Filippo M, Portaccio E, Mancini A, et al. Multiple sclerosis and cognition: synaptic failure and network dysfunction. Nat Rev Neurosci 2018;19:599-609.

69 Hardmeier M, Leocani L, Fuhr P. A new role for evoked potentials in MS? repurposing evoked potentials as biomarkers for clinical trials in MS. Mult Scler 2017;23:1309-19.

70 Gschwind M, Hardmeier M, Van De Ville D, et al. Fluctuations of spontaneous EEG topographies predict disease state in relapsing-remitting multiple sclerosis. Neuroimage Clin 2016;12:466-77.

71 Pokryszko-Dragan A, Bilinska M, Gruszka E, et al. Assessment of visual and auditory evoked potentials in multiple sclerosis patients with and without fatigue. Neurol SCi 2015:36:235-42.

72 Kale N, Agaoglu J, Onder G, et al. Correlation between disability and transcranial magnetic stimulation abnormalities in patients with multiple sclerosis. J Clin Neurosci 2009;16:1439-42.

73 Krieger SC, Cook K, De Nino S, et al. The topographical model of multiple sclerosis. Neurol Neuroimmunol Neuroinflamm 2016;3.

74 Farzan F, Atluri S, Frehlich M, et al. Standardization of electroencephalography for multi-site, multi-platform and multi-investigator studies: insights from the Canadian biomarker integration network in depression. Sci Rep 2017;7.

75 Snyder SM, Rugino TA, Hornig M, et al. Integration of an EEG biomarker with a clinician's ADHD evaluation. Brain Behav 2015;5:e00330.

76 Sanacora G, Smith MA, Pathak S, et al. Lanicemine: a low-trapping NMDA channel blocker produces sustained antidepressant efficacy with minimal psychotomimetic adverse effects. Mol Psychiatry 2014;19:978-85.

77 Scheltens P, Twisk JWR, Blesa R, et al. Efficacy of Souvenaid in mild Alzheimer's disease: results from a randomized, controlled trial. JAD 2012;31:225-36.

78 Ljubisavljevic MR, Ismail FY, Filipovic S. Transcranial magnetic stimulation of degenerating brain: a comparison of normal aging, Alzheimer's, Parkinson's and Huntington's disease. Curr Alzheimer Res 2013;10:578-96.

79 Tsolaki A, Kazis D, Kompatsiaris I, et al. Electroencephalogram and Alzheimer's disease: clinical and research approaches. Int J Alzheimers Dis 2014:2014. 